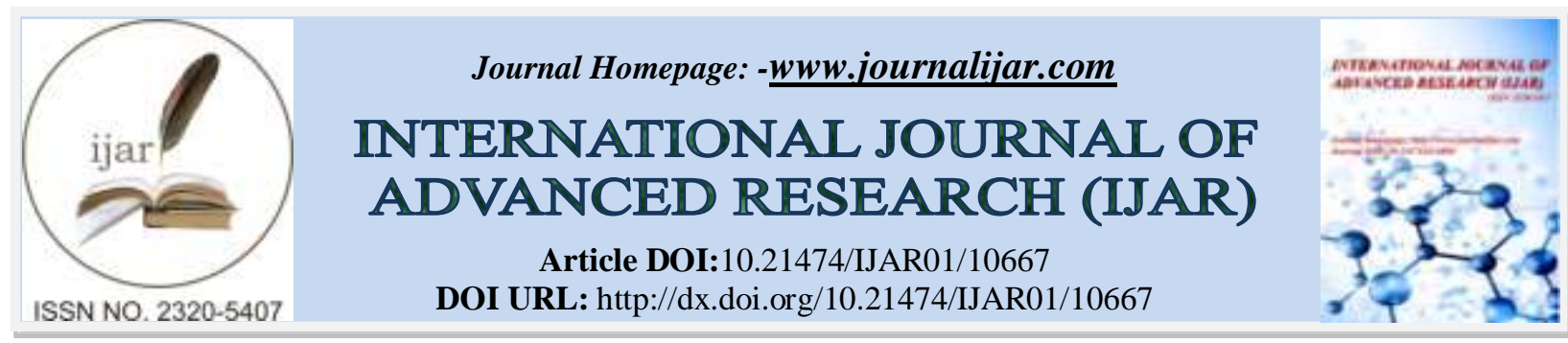

RESEARCH ARTICLE

\title{
UNUSUAL BEHAVIOR OF RADICULAR CYST IN PREMAXILLA _ A CASE REPORT
}

Mona Nady Abd El-Raouf, Kirolos Makram Hana and Abdul Motakaber Galal

Resident, Department of Oral and Maxillofacial Surgery, Faculty of Dentistry, 6 October University, Giza, Egypt.

\section{Manuscript Info}

\section{Manuscript History}

Received: 15 January 2020

Final Accepted: 17 February 2020

Published: March 2020

Key words:-

Radicular Cyst, Inflammatory Cyst,

Premaxilla, Maxilla

\begin{abstract}
Radicular cysts are the most common odontogenic cysts of inflammatory origin affecting tooth bearing area of the maxillofacial origin with ratio of $52 \%$ to $68 \%$. Its clinical picture more or less similar to many other common lesions of oral cavity, it has symptomless growth pattern usually slow growing unless it becomes infected or enlarged.Mobility; displacement; and root resorption of the adjacent teeth are common finding. It's more common in males with peak in third to fourth decade. Majority occur in maxilla with incidence of $60 \%$. Radiographic most of these lesions are well-defined radiolucency. The present case report represent a large radicular cyst involving premaxilla with extension to maxillary sinus and crossing the midline of the palate. The lesion was surgically enucleated along with endodontic treatment and apicectomy of involved teeth.
\end{abstract}

Copy Right, IJAR, 2020,. All rights reserved.

\section{Introduction:-}

Odontogenic cysts mainly classified according to who classification into developmental and inflammatory types in base of their etiology. Inflammatory odontogenic cysts include the radicular cysts and the paradental cysts. Radicular cysts (periapical cyst, lateral periodontal cyst, residual cyst) are the most common inflammatory odontogenic cysts of tooth bearing areas of the jaws.[1][2] They formed by inflammation of periodontal ligament of involved teeth. They are mostly related to pulpal affected teeth; meanwhile, they sometimes found related to accessory root canals.

Clinically, radicular cysts more common present between the third and the sixth decade of life with male predilection. The lesion may exhibit mild pain and sensitivity to percussion. Usually present with non-vital tooth and bluish discoloration of surround mucosa.

Radiographic, round or ovoid uni- or multilocular radiolucency with radiopaque margin arise from lamina dura of involved tooth. Although, this margin disappears with inflammation and rapid extension. In this article, we present a case of giant, aggressive radicular cyst of premaxilla invading the entire right maxillary sinus and crossing the midline of palate. [3]

\section{Case Report:-}

26-year-old male patient reported to maxillofacial outpatient clinic (faculty of dentistry -6 October University) with a chief symptom of mild pain on biting related to the left upper anterior teeth region. The dental history revealed past dental trauma in young age without history of seeking dental care with repeated prescription of antibiotics and analgesics at variant dental clinics for the same persistent swelling since 6 months. Medical history was free of any 
compromised disease with history of past 10 years of heavy smoking. Upon clinical examination, buccal and palatal swelling extending from upper right central to upper left canine, crossing the midline of palate (Figure 1). There were crown fracture in relation to both upper central and upper right lateral, which were non-vital. The adjacent teeth were tested vital. On palpation, the lesion was assumed egg shell cracking. The buccal plate was perforated with no present draining sinus tract. No mobility or change in occlusion present. Extra oral head and neck examination was unremarkable. There was no evidence of lymphadenopathy or any abnormal masses or rashes. Oral hygiene was poor with heavy stains and calculus. Radiographic examination revealed a large hypodensity with welldefined radiopaque border (Figure 2). The lesion was approximately $2.5 \times 2 \times 4 \mathrm{~cm}$. Routine laboratory investigations were within normal limits. Needle aspiration revealed turbid brown-colored fluid.

\section{Differential diagnosis:}

According to clinical and radiographic characteristics, the differential diagnosis of the radicular cysts should include dentigerous cyst, pindborg tumour, traumatic bone cyst, ameloblastoma, odontogenic keratocyst, and odontogenic fibroma. Confirmatory diagnosis of the radicular cyst is established only after surgical biopsy and histopathological examination of the lesion. [4]

\section{Treatment:}

Performed under general anesthesia, a crevicular incision from upper left incisor to upper right first premolar with anterior releasing mucosa was placed and a full mucoperiosteal flap was raised. Involved teeth were endodontic treated and apicectomy performed. A plane of cleavage was established between cystic epithelial lining and surrounding bone (Figure 3). The whole cystic lining measured (2.3) was enucleated into and it was sent for histopathological examination and curettage was done to make sure all remnants were removed; lesion was found to be eroding the medial and posterior wall of sinus, with feature of secondary infection with discharge of brown cheesy material. Primary closure of cystic cavity done with vicryl 3-0 sling sutures placed through interdental papilla, and interrupted sutures over releasing incision (Figure 4).Microscopic examination of biopsy revealed histological features of infectedradicular cyst. The patient was kept on regular follow-up. The wound healed uneventfully.

\section{Discussion:-}

Radicular cysts has wide age range, however, they are rarely seen in children regardless of high incidence of pathological involvement of pulp in this category, which may be caused byabsence of epithelial remnant related to primary teeth.

Clinically, these cysts are usually associated with dental history of a carious tooth that is carious or subjected to restorative treatment, trauma, or failure of root canal therapy. Radiographically, usually present as small apical radiolucency, rare will be bony expansion if secondary infected or enlarged.

Prognosis, resolve if the tooth and the apical lesion are removed. If tooth is removed and the cyst is not, most cysts will involute because of the removal of the inflammatory focus. A few rare cases will retain their cystic stimulation independent of the tooth, probably by ongoing inflammation in the wall of the cyst. This is termed a residual cyst. Endodontically treated teeth will resolve radicular cysts as long as they have a successful pulp canal debridement and fill. Apicoectomies with a retrofill of the apical area and curettage of the residual lesion or tooth removal is indicated if this retreatment fails. [5][4][6]

\section{Conclusion:-}

Periodic clinical and radiographic dental examination can aid early detection of pathological lesion and prevent further destruction of bony component of jaws .Even little source of inflammation like exposed tooth or previous trauma can cause large pathological destruction of jaws upon histological and radiographic analysis of such lesions. 


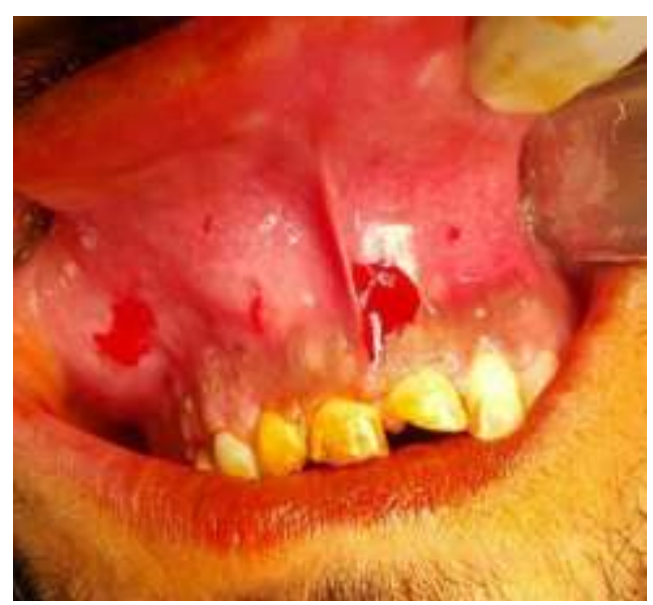

Figure 1:- Pre-operative photograph reveal intra oral swelling.

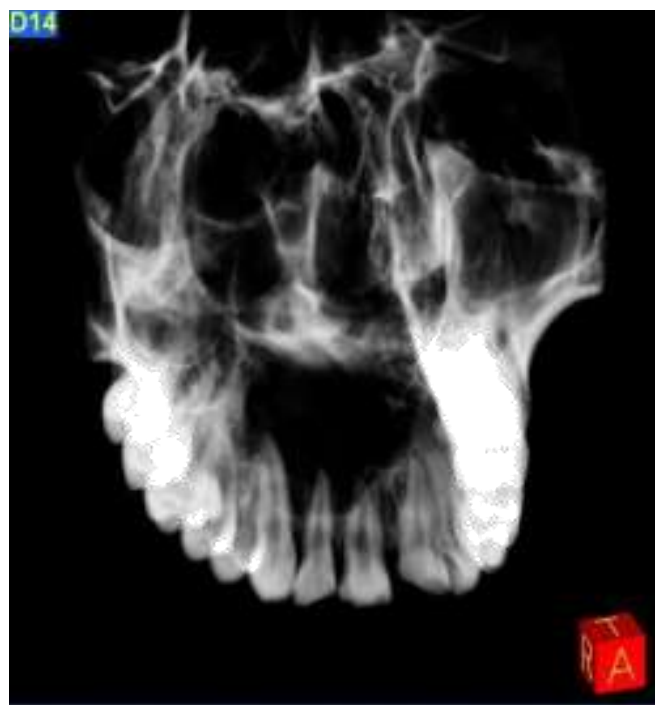

Figure 2:- CBCT showing well defined hypodensity in bone related upper anterior teeth.

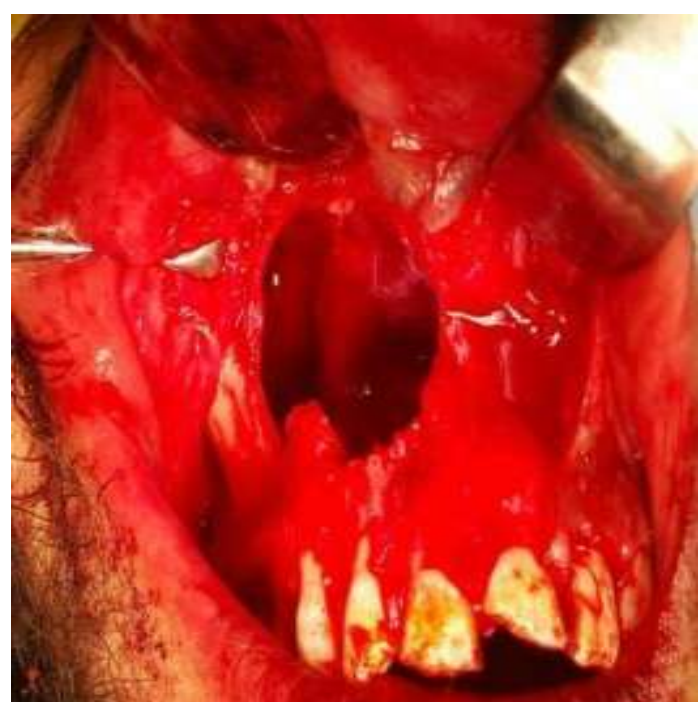

Figure 3:- Intra operative photograph after enculation of lesion. 


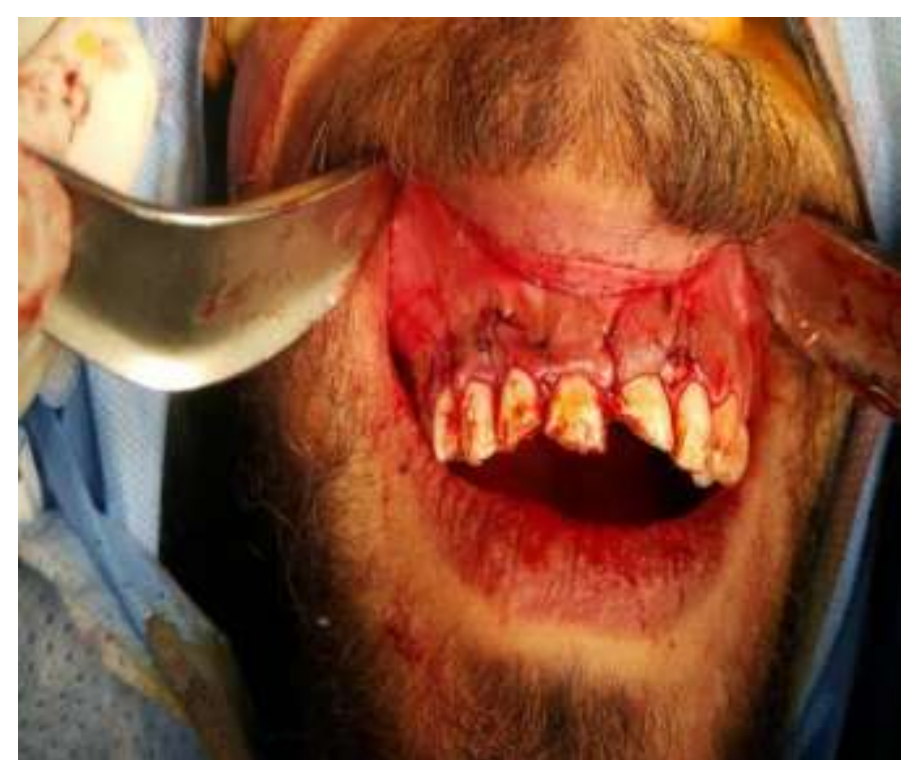

Figure 4:- Photograph after primary closure.

\section{References:-}

1. V. Krishnamurthy, "Radicular Cyst Masquerading as a Multilocular Radiolucency," Quintessence Int, 2013.

2. N. S. Kadam, "Management of Large Radicular Cyst by Conservative Surgical Approach: A Case Report," J Clin Diagn Res, 2014.

3. Quadri, "Unusual Aggressive Large Radicular Cyst Invading Maxillary Sinus: A Case Report," IJSS, 2006.

4. S. M., Radicular and residual cysts. In: Cysts of the Oral Region, 1992.

5. Odontogenic Cystsand Tumors in Oral and Maxillofacial Pathology..

6. Oral Pathology. 5th ed., New Delhi:: Elsevier, 2006. 